\title{
Incidence of Acute Kidney Injury Among Cases with Suicidal Attempts of Paraphenylenediamine Poisoning in Aswan University Hospital Omaima Mohamed Ali ${ }^{1}$, Lalaa Abd Elrahem Othman ${ }^{1}$, Wafaa Salah Mohammed ${ }^{2}$, Wael Abd Elgwad Elsewify ${ }^{1 *}$ \\ Departments of ${ }^{1}$ Internal Medicine and ${ }^{2}$ Clinical Pathology, Faculty of Medicine, Aswan University, Aswan, Egypt \\ *Corresponding author: Wael Abd Elgwad Elsewify, Department of Internal Medicine, Faculty of Medicine, Aswan University, Aswan, Egypt, Tel: +201001657295, Email: waelelsewify@ yahoo.com
}

\begin{abstract}
Background: The rate of suicidal attempts with paraphenylenediamine (PPD) poisoning has raised steadily in the past few decades. Recently, a growing body of evidence demonstrated that PPD can lead to acute kidney injury (AKI).

Objectives: To assess the incidence of AKI among cases with suicidal attempts of PPD poisoning in Aswan University hospital.

Patients and Methods: Our study was a cross-sectional study that included 94 patients presented with suicidal attempts of paraphenylenediamine (dark hair dye) at Aswan University Hospital during the period from January to December 2019. All of the participants were subjected to history taking as age, sex, route of administration, suicidal or accidental intake of dye, type and amount of dye used, and duration of intake. Examination and investigations were as follows, vital data: BP, temperature, pulse, respiratory rate, electrocardiogram (ECG ), urine analysis, blood urea, serum creatinine, serum sodium, serum potassium, creatine phosphokinase (CPK), alkaline phosphatase, alanine aminotransferase (ALT), aspartate aminotransferase (AST), serum uric acid, arterial blood gases $(\mathrm{ABG})$ and follow up for the development of any complications or any interventions as tracheostomy, intubation, and hemodialysis.
\end{abstract}

Results: The mean age of the included patients was $26.1 \pm 12.9$ years and the majority of them were females $(66 \%)$. In terms of the primary outcome of the present study, we found that the incidence of AKI was $29.7 \%$. There were statistically significant differences between AKI and non-AKI patients in terms of serum ALT (p $=0.001)$, serum AST $(p=0.001)$, serum urea $(p=0.001)$, serum creatinine $(p=0.001)$, and serum CPK $(p=0.001)$. Additionally, patients with AKI had a significantly higher mortality rate than non-AKI patients $(\mathrm{p}=0.023)$. Serum CPK was a major predictor of AKI in the univariate analysis.

Conclusion: In conclusion, AKI is considered one of the most common problems in patients with suicidal attempts of PDD ingestion. AKI is one of the leading causes of mortality in patients with PPD ingestion.

Keywords: AKI, Paraphenylenediamine, Suicidal attempt.

\section{INTRODUCTION}

Suicide is a main public health concern in both developing and developed countries, which contribute to at least $2 \%$ of global health issues ${ }^{(\mathbf{1})}$. According to the World Health Organization (WHO) report, it is estimated that suicide will kill one and a half million people worldwide by the end of $2020^{(2)}$. Over the past two decades, the rate of suicidal attempts has increased exponentially in developing countries, recent reports estimated that more than two-thirds of the global suicidal attempts occur in developing countries alone ${ }^{(3)}$.

Oral ingestion of paraphenylenediamine (PPD) is a common suicidal method, especially in the Middle East and East Africa. PPD is an aniline derivative commonly present in commercial hair dye formulations, it presents in henna (Lawsonia alba) to darken its color, as well as in many-body tattooing materials ${ }^{(4)}$. According to a recently updated metaanalysis study, the prevalence of suicidal attempts in patients present with PPD poisoning is $93 \%{ }^{(5)}$. The systematic administration of PDD can lead to a variable degree of edema in various body parts that can extend to larynx leading to suffocation and respiratory distress. Besides, PPD ingestion can be complicated by rhabdomyolysis, acute hepatitis, acute renal failure, myocarditis, arrhythmias, metabolic abnormalities, seizures, shock, and eventually death ${ }^{(6)}$. Moreover, survivors can suffer from long-term sequelae such as chronic kidney disease requiring maintenance hemodialysis ${ }^{(7)}$.

The direct toxic effects of PPD can lead to many pathological problems. Therefore, patients with PPD poisoning can present with some hematological and biochemical abnormalities ${ }^{(8)}$.

Hair dye ingestion is a medical emergency. Emergency measures should include gastric lavage. Patients should be monitored for respiratory distress and endotracheal intubation has to be performed early if laryngeal edema develops. Metabolic 
acidosis has to be corrected. Rhabdomyolysis can be treated with half normal saline. Acute renal failure as a result of PPD poisoning can be managed by hemodialysis, peritoneal dialysis, and continuous renal replacement therapy and it was found to be beneficial. Mortality rates vary between $0.03 \%$ and $60 \%{ }^{(9)}$.

On the other hand, acute kidney injury (AKI) has been recognized as a serious and common disorder with a wide range of complications. AKI is a condition in which there is a rapid, marked, deterioration of renal function, which is usually reversible ${ }^{(10)}$. A large dose of PPD can lead to death, actually not known but it can vary from 7-10 gram (11). PPD is poisonous when ingested and the higher the dose the worse is the outcome. The large dose of PPD can induce fatal cardiac arrhythmia ${ }^{(10)}$.

Recently, a growing body of evidence demonstrated that PPD poisoning can lead to AKI. However, the published literature is scarce regarding the prevalence of AKI among PPD poisoning cases in Egypt. Thus, we conducted the present study to assess the incidence of AKI among cases with suicidal attempts of PPD poisoning in Aswan University Hospital.

\section{AIM OF THE WORK}

The study aims to determine and investigate how much PPD can affect kidney in patients taking dark hair dye in Aswan University Hospital.

\section{PATIENTS AND METHODS}

\section{Study design:}

Our study was a cross-sectional study, we retrieved the data of all patients presented with a suicidal attempt by oral ingestion of PDD to Aswan University Hospital through the period from January to December 2019. Patients $\geq 14$ years old of both sexes were included in our study. Patients with a history of CKD, prior history of PPD injection, history of nephrotoxic drug exposure, acute hepatitis, chronic liver disease, cirrhosis, and/or missing follow-up data were excluded.

\section{Data Collection:}

We collected the following data from each eligible patient: demographic characteristics, clinical features, liver function tests, serum urea, serum creatinine, serum creatine phosphokinase (CPK), serum electrolytes, needs for tracheostomy, needs for hemodialysis, occurrence complications, prognosis, death, and incidence of AKI. The definition of AKI was based on the criteria of Kidney Disease Improving Global Outcomes (KDIGO) classification ${ }^{(\mathbf{1 2})}$. Thus, the AKI was diagnosed if the elevation of serum creatinine reached $\geq 0.3 \mathrm{mg} / \mathrm{dL}$ in two days or one-and-a-half-fold increase from baseline in one week.

\section{Ethical Statement:}

We confirm that this study is consistent with international ethical standards and the applicable local regulatory guidelines. The study has no physical, psychological, social, legal, economic, or other expected risks to the study participants. Participants in the study were informed of objectives, methods, risks, and benefits. Written informed consent has been obtained from every qualifying patient in the study. Our study was conducted under the Declaration of Helsinki for studies on human subjects. It was approved by the institutional review board of The Faculty of Medicine, Aswan University.

\section{Statistical Analysis}

The analyses were done by SPSS software (Statistical Package for Social Sciences, version 24, SSPS Inc, Chicago, IL, USA). Numerical data were described as mean \pm SD or median and interquartile range $[\mathrm{IQR}]$. Frequency tables were used for categorical variables. Independent Student t-test and ANOVA tests were used to compare parametric quantitative variables; while Mann-Whitney tests and Kruskal Wallis tests were used to compare nonparametric quantitative variables. A Chi-square test was used to analyze categorical variables. A pvalue $<0.05$ is considered statistically significant.

\section{RESULTS}

The present study was conducted on 94 patients presented with suicidal attempts of PPD at Aswan University Hospital during the period from January to December 2019. The mean age of the included patients was $26.1 \pm 12.9$ years and the majority of them were females (66\%). The most common presentation was cervicofacial edema $(85.1 \%)$, followed by difficulty in opening the mouth (73.4\%), toxic hepatitis (55.3\%), and dark urine (50\%). The mean serum urea and creatinine of the included patients were $69.4 \pm 60.1$ and $2.1 \pm 1.6$ $\mathrm{mg} / \mathrm{dL}$, respectively. Also, the averages serum AST and ALT were $433.1 \pm 987.1$ and $353.5 \pm 60.1 .1$, respectively. The mean serum CPK was 3380.2 \pm 13120.3 U/L (Table 1). 
Table (1): The basic data of the study population

\begin{tabular}{|l|l|c|}
\hline \multicolumn{1}{|c|}{ Variables } & Patients (N =94) \\
\hline \multirow{3}{*}{ Gge in years } & Mean \pm SD & $26.1 \pm 12.9$ \\
& Median (range) & $22(15-25)$ \\
\hline \multirow{5}{*}{ Clinical Features, No. (\%) } & Female & $62(66 \%)$ \\
\cline { 2 - 3 } & Male & $32(34 \%)$ \\
& Cervicofacial edema & $80(85.1 \%)$ \\
& Difficulty in opening the mouth & $69(73.4 \%)$ \\
& Muscle weakness & $33(35.2 \%)$ \\
& Dark urine & $47(50 \%)$ \\
& Seizures & $2(2.2 \%)$ \\
& Acidosis & $30(31.9 \%)$ \\
& Hepatitis & $52(55.3 \%)$ \\
\hline \multirow{5}{*}{} & Serum ALT (IU/L) & $60(17-6800)$ \\
\cline { 2 - 3 } & Serum AST (IU/L) & $75(16-2800)$ \\
\cline { 2 - 3 } & Serum Urea (mg/dL) & $35(15-219)$ \\
\cline { 2 - 3 } & Serum creatinine (mg/dL) & $1.2(0.5-6)$ \\
\cline { 2 - 3 } & Creatine Phosphokinase (U/L) & $450(50-90000)$ \\
\cline { 2 - 3 } & Na level (mmol/L) & $136(133-153)$ \\
\cline { 2 - 3 } & K level(mmol/L) & $4.1(3.1-6.4)$ \\
\hline
\end{tabular}

AST, aspartate aminotransferase; ALT, alanine aminotransferase; CKP, creatine phosphokinase; Na, serum sodium; K, serum potassium

Thirty-seven patients (39.4\%) required tracheostomy. Also, $16 \%$ of the patients received hemodialysis. The prognosis was normal in $25.5 \%$ of the patient. On the other hand, the most common complications were laryngeal edema (28.7\%), elevated Liver enzyme (25.5\%), ventricular arrhythmias (20.2\%), myocarditis (10.6\%), sepsis (9.6\%), and ESRD (7.4\%). Twenty-six patients (27.7\%) died during hospitalization. The most common causes of death were respiratory distress, myocarditis, fatal arrhythmia, and sepsis.

Table (2): The prognosis of the study population

\begin{tabular}{|c|c|c|}
\hline  & Variables & Patients (N =94) \\
\hline Tracheostomy, No. (\%) & Yes & $37(39.4 \%)$ \\
\hline \multirow[t]{2}{*}{ Hemodialysis, No. (\%) } & $\begin{array}{l}\text { No } \\
11 \text { Sessions } \\
3 \text { Sessions /week for } 3 \text { months } \\
5 \text { Sessions } \\
7 \text { Sessions } \\
8 \text { Sessions }\end{array}$ & $\begin{array}{l}79(84 \%) \\
3(3.2 \%) \\
4(4.3 \%) \\
4(4.3 \%) \\
2(2.1 \%) \\
2(2.1 \%) \\
\end{array}$ \\
\hline & $\begin{array}{l}\text { Prognosis, No. (\%) } \\
\text { Pass normal } \\
\text { Complicated }\end{array}$ & $\begin{array}{l}24(25.5 \%) \\
70(74.5 \%)\end{array}$ \\
\hline Type of complication, No. (\%) & $\begin{array}{l}\text { Laryngeal edema } \\
\text { Elevated Liver enzymes } \\
\text { Hyperkalemia } \\
\text { Myocarditis } \\
\text { Ventricular arrhythmias } \\
\text { Sepsis } \\
\text { CKD and/or ESRD }\end{array}$ & $\begin{array}{c}27(28.7 \%) \\
24(2.1 \%) \\
6(6.4 \%) \\
10(10.6 \%) \\
19(20.2 \%) \\
9(9.6 \%) \\
7(7.4 \%)\end{array}$ \\
\hline \multirow[t]{2}{*}{ Mortality, No. (\%) } & Died & $26(27.7 \%)$ \\
\hline & Alive & $68(72.3 \%)$ \\
\hline \multirow{4}{*}{ Cause of Death, No. (\%) } & Respiratory distress & $8(8.5 \%)$ \\
\hline & Myocarditis or fatal arrhythmia & $7(7.5 \%)$ \\
\hline & Sepsis after hospitalization & $7(7.5 \%)$ \\
\hline & Acute renal failure. & $4(4.3 \%)$ \\
\hline
\end{tabular}

Our study demonstrated that by univariate analysis that serum CPK was the most important predictor of AKI and mortality in studied patients (Table 3 and 4). 
Table (3): Univariate Analysis of Predictors of AKI.

\begin{tabular}{|l|c|c|}
\hline \multicolumn{1}{|c|}{ Variables } & \multicolumn{2}{c|}{ Univariate Analysis } \\
\hline Age in years & B Coefficient & P- Value \\
\hline Male Gender & 0.101 & 0.47 \\
\hline Serum ALT & -0.296 & 0.014 \\
\hline Serum AST & 0.597 & 0.57 \\
\hline Serum CPK & 0.654 & 0.72 \\
\hline Serum Sodium & 0.887 & 0.035 \\
\hline Serum potassium & 0.229 & 0.27 \\
\hline
\end{tabular}

AST, aspartate aminotransferase; ALT, alanine aminotransferase; CKP, creatine phosphokinase. *Data are presented as a correlation coefficient.

Table (4): Univariate Analysis of Predictors of Morality.

\begin{tabular}{|l|c|c|}
\hline & \multicolumn{2}{|c|}{ Univariate Analysis } \\
\hline Variables & B Coefficient & P- Value \\
\hline Age in years & -0.242 & 0.04 \\
\hline Male Gender & -0.296 & 0.014 \\
\hline Serum ALT & 0.01 & 0.94 \\
\hline Serum AST & 0.01 & 0.93 \\
\hline Serum CPK & -0.324 & 0.006 \\
\hline
\end{tabular}

AST, aspartate aminotransferase; ALT, alanine aminotransferase; CKP, creatine phosphokinase. *Data are presented as a correlation coefficient

Our study results showed that AKI of the included patients was presented in twenty-eight patients (29.7\%). There were no statistically significant differences between AKI and non-AKI patients in terms of age $(\mathrm{p}=0.64)$ and $\operatorname{sex}(\mathrm{p}=0.09)$. Likewise, there were no statistically significant differences between AKI and nonAKI patients in terms of Na level $(p=0.17)$ and $\mathrm{K}$ level $(\mathrm{p}=0.15)$. On the contrary, patients with AKI had significantly higher serum ALT $(p=0.001)$, serum AST $(p=0.001)$, serum urea $(p=0.001)$, serum creatinine $(p$ $=0.001)$, and serum CPK $(\mathrm{p}=0.001)$. Moreover, patients with AKI had a significantly higher mortality rate $(\mathrm{p}$ $=0.023)$. Besides, there was no statistically significant difference between AKI and non-AKI patients in terms of tracheostomy $(\mathrm{p}=0.65)$ (Table 5).

Table (5): Association between AKI and Different Parameters

\begin{tabular}{|c|c|c|c|c|}
\hline & Variables & AKI (N =28) & No AKI $(\mathbf{N}=66)$ & P-value \\
\hline Age in years & Mean \pm SD & $25.18 \pm 12.1$ & $26.48 \pm 13.4$ & 0.64 \\
\hline \multirow[t]{2}{*}{ Gender, No. (\%) } & Male & $6(21.4 \%)$ & $26(39.4 \%)$ & 0.09 \\
\hline & Female & $22(78.6 \%)$ & $40(60.6 \%)$ & \\
\hline Serum ALT (IU/L) & - Mean \pm SD & $850.86 \pm 1587$ & $255.95 \pm 464$ & 0.805 \\
\hline Serum AST (IU/L) & - Mean \pm SD & $2.87 \pm 0.9$ & $1.7 \pm 0.7$ & 0.183 \\
\hline Serum Urea (mg/dL) & - Mean \pm SD & $92.64 \pm 37.6$ & $59.54 \pm 65.1$ & 0.001 \\
\hline Serum creatinine (mg/dL) & - Mean \pm SD & $2.87 \pm 0.9$ & $1.7 \pm 0.7$ & 0.001 \\
\hline Serum CPK (U/L) & - Mean \pm SD & $9420 \pm 23133$ & $817.88 \pm 1270$ & 0.001 \\
\hline Na level $(\mathrm{mmol} / \mathrm{L})$ & - Mean \pm SD & $136.1 \pm 1.9$ & $137.1 \pm 3.7$ & 0.17 \\
\hline K level (mmol/L) & - Mean \pm SD & $4.57 \pm 0.76$ & $4.3 \pm 0.87$ & 0.15 \\
\hline Tracheostomy, No. (\%) & $\begin{array}{l}\text { Yes } \\
\text { No }\end{array}$ & $\begin{array}{l}12(42.8 \%) \\
16(57.2 \%)\end{array}$ & $\begin{array}{l}25(37.9 \%) \\
41(62.1 \%)\end{array}$ & 0.65 \\
\hline Mortality, No. (\%) & $\begin{array}{l}\text { Yes } \\
\text { No }\end{array}$ & $\begin{array}{l}12(42.8 \%) \\
16(57.2 \%)\end{array}$ & $\begin{array}{l}14(21.2 \%) \\
52(78.8 \%)\end{array}$ & 0.023 \\
\hline
\end{tabular}

AST, aspartate aminotransferase; ALT, alanine aminotransferase; CKP, creatine phosphokinase; Na, serum sodium; K, serum potassium. 
Paraphenylenediamine (PPD) poisoning is emerging as an important etiological factor for suicidal attempts in developing countries. PPD poisoning was the first cause of poisoning in many countries in the early 2000s including the North Africa region. Several reports have explained the fatal ingestion of hair dye containing PPD ${ }^{(5)}$.

On the other hand, acute kidney injury (AKI), previously seen predominantly as a self- limited and reversible disease, is now recognized as a growing issue associated with substantial long-term adverse health risks. Many studies have documented a significant relationship between AKI and subsequent risks of AKI recurrence, hospital re-entry, cardiovascular diseases, and cancer morbidity and mortality, as well as CKD and ESRD development (13).

Recently, a growing body of evidence demonstrated that PPD poisoning can lead to AKI. However, the published literature is scarce regarding the prevalence of AKI among PPD poisoning cases in Egypt. In the present study, we found that the incidence of AKI among PPD poising cases was 29.7\%. Elevated serum ALT, serum AST, serum urea, serum creatinine, and serum CPK at presentation appear to be predictors of AKI. On the other hand, AKI is one of the predictors of mortality in PPD poisoning cases.

The occurrence of AKI among cases of PPD ingestion appears to be multifactorial, the most common theory for AKI in PPD cases assumes that the AKI results from direct renal damage by the toxic agent itself, hypovolemic shock, hemolysis and rhabdomyolysis ${ }^{(7)}$. In terms of the present study, we found that the incidence of AKI was 29.7\%. In agreement with our findings, Kallel et al. (14) reported that the incidence of AKI was $26.3 \%$. Another report by Sanchez et al. ${ }^{(15)}$ found that the incidence of AKI was $21.2 \%$.

However, other studies reported higher rates of AKI than our results. Abdelraheem et al. ${ }^{(16)} \mathrm{did}$ their study to estimate clinical manifestations, management, and outcome of PPD intoxication in Sudanese children in the period between 2006 to 2008. The study included 17 children. Data was reported from the Paediatric Nephrology Unit, Soba University Hospital. Six-teen females were admitted with PPD intoxication, of the 12 children $(71 \%)$ developed ARF, nine were in need for dialysis, and three were treated conservatively. Two children died (12\%), and the other 15 recovered with normal renal function.

Similarly, Bhagavathula et al. ${ }^{(5)}$ reported that acute renal failure was noticed in $54.7 \%(95 \% \mathrm{CI}=$ 34.5-74.9) of the pooled samples and mortality rates were $14.5 \%$ (95\% CI $=11.1-17.9)$. Suliman et al. (17) reviewed 150 cases presenting to Khartoum
Teaching Hospital with PPD poisoning for 10 years, with regards to the renal involvement, 90 of the 150 study cases $(60 \%)$ developed acute renal failure (ARF) requiring dialysis.

The exact causes of such difference between our findings and the above-mentioned studies are not clear. However, this difference can be explained by the difference in sample size or the definition of AKI which may be another cause.

PPD poisoning poses a considerable risk with mortality rates ranging from $12 \%$ to $42 \%{ }^{(18)}$. In the present study, we found that 26 patients $(27.7 \%)$ died during hospitalization. The most common causes of death were respiratory distress, myocarditis, fatal arrhythmia, sepsis, and AKI.

In concordance with our findings, Kallel et al. (14) reported the clinical symptoms and outcome of systemic PPD intoxication. The study was retrospective. It was conducted over 6 years (19942000) in the medical intensive care unit (ICU) of a university hospital and it concerned 19 hospitalized patients for systemic PPD intoxication. The intoxication evolution was marked by the death of six patients (31.6\%); five of them had developed ARF. Similarly, Ishtiaq et al. ${ }^{(19)}$ reviewed the medical records of 109 patients with PPD intoxication admitted to Medical Unit-2, Bahawalpur Victoria Hospital from January 2015 to June 2015, in a descriptive, cross-sectional study. Overall mortality was noted in 39 patients (36\%). Yagi et al. ${ }^{(20)}$ reported the first series of 18 cases with acute hair dye (PPD) poisoning over two years. Poisoning was with suicidal intent in $70 \%$ of the cases and accidental in other cases. Sixteen cases were classified as serious requiring tracheostomy. Four out of 18 patients died with a mortality rate of $22 \%$.

Similar to our findings, Haider et al. (21) conducted an observational prospective analytic study that included 32 patients who presented in the ICU of Nishtar Hospital, Multan. Tracheostomy was required in 30 patients (93.7\%), and 13 (40.6\%) patients required mechanical ventilation support.

Likewise, Tanweer et al. (22) aimed to determine the clinical presentation, complications, and outcome of PPD poisoning in patients presenting to Nishtar Hospital, Multan. All adult patients admitted with a history of PPD ingestion were evaluated. Tracheostomy was done in 95/116 (82\%) patients with cervicofacial edema. Myocarditis as a complication was diagnosed in 33/122 (27\%) patients.

Nonetheless, other reports demonstrated a higher or lower mortality rate than our report. For example, Khaskheli et al. ${ }^{(23)}$ reported that the mortality rate in 993 patients was $14.5 \%(\mathrm{n}=139)$ in males and $17.52 \%(n=172)$ in females. While Soni 
et al. (24) included, between January 2006 and February 2007, 10 patients (6 males, 4 females) of PPD (3.75\%) ingestion in the form of hair dye, with suicidal intent. Six of the 10 patients died (mortality, $60 \%)$.

Umair et al. ${ }^{(6)}$ reported a case report of a young female with PPD ingestion, her condition deteriorated in terms of cervicofacial edema and elevation of CPK level which is in agreement with our study.

The exact causes of such difference between our findings and the above studies are not known. However, this difference can be explained by the variations in the characteristics and demography of the included patients; as well as the method of assessment. The difference in sample size may be another cause.

\section{CONCLUSION}

In conclusion, AKI is one of the most common problems in patients with suicidal attempts of PDD ingestion. Elevated serum $\mathrm{CPK}$ at presentation appears to be a strong predictor of AKI. On the other hand, AKI is one of the major predictors of mortality in PPD poisoning cases. Further studies are warranted for a better understanding of the underlying pathogenic mechanism of AKI in PPD poisoning.

Disclosure: The authors declare that they have no competing interests.

\section{REFERENCES}

1. World Health Organization. (2016): Suicide: Fact sheet. World Health Organization. https://www.who.int/health-topics/suicide.

2. World Health Organization (2001): The World Health Report: Mental Health Report: New understanding, new hope.www.who.int $>$ whr $>2001$

3. Demissie, Z, Clayton HB (2018): Prevalence of Suicide Ideation, Planning, and Attempts among Adolescents in 83 Developing Countries, 2003-2016. Journal of Adolescent Health, 62(2): 15-18.

4. Akl A, Alturki RM (2018): Paraphenylenediamine hair dyeing nephropathy: a case report and review of the literature. Urology \& Nephrology Open Access Journal, 6(5): 147-149.

5. Bhagavathula AS, Bandari DK, Khan M et al. (2019): A systematic review and meta-analysis of the prevalence and complications of paraphenylenediamine-containing hair dye poisoning in developing countries. Indian Journal of Pharmacology, 51(5): 302-315.

6. Umair SF, Amin I, Ur Rehman A (2018): Hair dye poisoning: "An early intervention." Pakistan Journal of Medical Sciences, 34(1): 230-232.

7. Shigidi M, Mohammed O, Ibrahim M et al. (2014): Clinical presentation, treatment, and outcome of paraphenylenediamine induced acute kidney injury following hair dye poisoning: A cohort study. Pan African Medical Journal, 19: 163-166.

8. Prabhakaran A (2012): Paraphenylenediamine poisoning. Indian J Pharmacol., 44:423-4.

9. Radhika D, Murali D, Sreenivasulu M et al. (2012): Hair Dye Poisoning-A Clinicopathological Approach and Review. J Biosci Tech., 3(4):492- 497.

10. Singbartl K, Joannidis M (2015): Short-term Effects of Acute Kidney Injury. Critical Care Clinics, 31(4):751-762.

11.Singla S, Miglani S, Lal AK et al. (2005): Paraphenylenediamine (PPD) poisoning. Journal Indian Academy of Clinical Medicine, 6(3): 236-238.

12. Kellum JA, Lameire N, Aspelin P et al. (2012): Kidney Disease: Improving Global Outcomes (KDIGO) Acute Kidney Injury Work Group. KDIGO Clinical Practice Guideline for Acute Kidney Injury. Kidney International, 2: 1-5.

13.James MT, Bhatt M, Pannu N et al. (2020): Longterm outcomes of acute kidney injury and strategies for improved care. Nature Reviews Nephrology. Nature Research, 16:193-205.

14. Kallel H, Chelly H, Dammak H et al. (2005) Clinical manifestations of systemic paraphenylenediamine intoxication. J Nephrol., 18:308-11.

15. Sanchez L, Handyal H, Kannan S et al. (2016): Hair dye poisoning: Retrospective analyses of patients admitted to ICU at a rural hospital in India. Indian J Med Res., 144:134-7.

16. Abdelraheem M, El-Tigani M, Hassan E et al. (2009): Acute renal failure owing to paraphenylenediamine hair dye poisoning in Sudanese children. Ann Trop Paediatr., 29:191-6.

17. Suliman S, Fadlalla M, Nasr M et al. (1995): Poisoning with hair-dye containing paraphenylenediamine: ten years experience. Saudi J Kidney Dis Transpl., 6:286-9.

18. Abdelraheem M, Hamdouk M, Zijlstra E (2010): Review: Paraphenylene Diamine (Hair Dye) Poisoning in Children. Arab J Nephrol Transplant., 3: 39-41.

19. Ishtiaq R, Shafiq S, Imran A et al. (2017): Frequency of Acute Hepatitis Following Acute Paraphenylene Diamine Intoxication. Cureus, 9(4):1186.

20. Yagi H, El Hind AM, Khalil SI (1991): Acute poisoning from hair dye. East Afr Med J., 68:404-11.

21. Haider SA, Sultan A, Salman Z et al. (2018): Paraphenylenediamine poisoning: Clinical presentations and outcomes. Anaesthesia, Pain Intensive Care, 22:43-7.

22. Tanweer S, Saeed M, Zaidi S et al. (2018): Clinical profile and outcome of paraphenylenediamine poisoning. J Coll Physicians Surg Pakistan, 28:374-7.

23. Khaskheli MS, Shaikh S, Meraj M et al. (2018): Paraphenylenediamine poisoning: Clinical features, complications and outcome in a tertiary care institute. Anaesthesia, Pain Intensive Care, 22:343-7.

24. Soni S, Nagarik A, Dinaker M et al. (2009): Systemic toxicity of paraphenylenediamine. Indian J Med Sci., 63:164-166. 